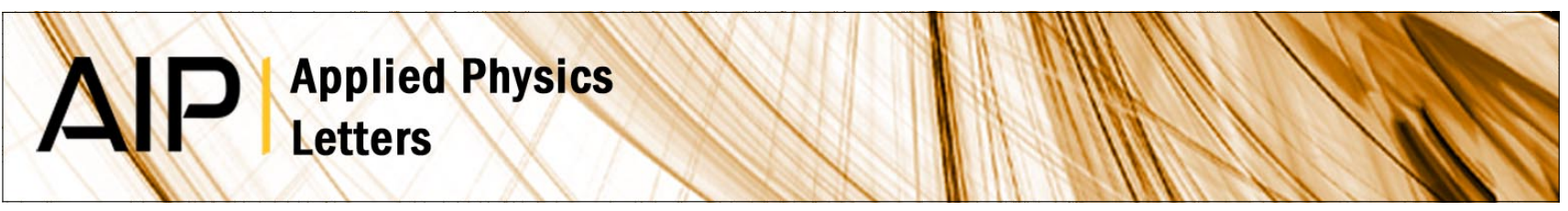

\title{
Microphotonic parabolic light directors fabricated by two-photon lithography
}

\author{
J. H. Atwater, P. Spinelli, E. Kosten, J. Parsons, C. Van Lare et al.
}

Citation: Appl. Phys. Lett. 99, 151113 (2011); doi: 10.1063/1.3648115

View online: http://dx.doi.org/10.1063/1.3648115

View Table of Contents: http://apl.aip.org/resource/1/APPLAB/v99/i15

Published by the American Institute of Physics.

\section{Related Articles}

Silicon carbide-based photonic crystal nanocavities for ultra-broadband operation from infrared to visible wavelengths

Appl. Phys. Lett. 99, 201102 (2011)

Simultaneous detection of surface coverage and structure of krypton films on gold by helium atom diffraction and quartz crystal microbalance techniques

Rev. Sci. Instrum. 82, 115104 (2011)

High-Q silica microcavities on a chip: From microtoroid to microsphere

Appl. Phys. Lett. 99, 181123 (2011)

Realization of negative refractive index with double-layered $\mathrm{H}$-shaped resonator array

Appl. Phys. Lett. 99, 181905 (2011)

One-way cloak based on nonreciprocal photonic crystal

Appl. Phys. Lett. 99, 151112 (2011)

\section{Additional information on Appl. Phys. Lett.}

Journal Homepage: http://apl.aip.org/

Journal Information: http://apl.aip.org/about/about_the_journal

Top downloads: http://apl.aip.org/features/most_downloaded

Information for Authors: http://apl.aip.org/authors

\section{ADVERTISEMENT}

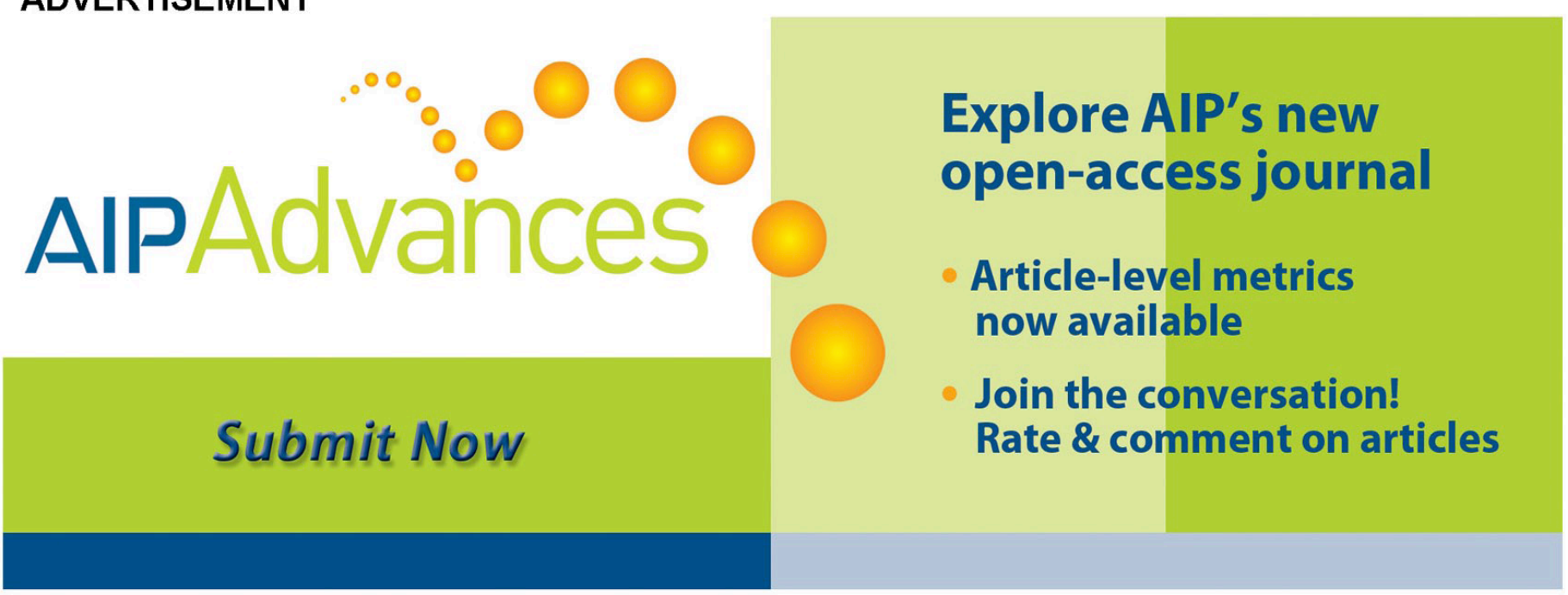




\title{
Microphotonic parabolic light directors fabricated by two-photon lithography
}

\author{
J. H. Atwater, ${ }^{1, a)}$ P. Spinelli, ${ }^{1}$ E. Kosten, ${ }^{2}$ J. Parsons, ${ }^{1}$ C. Van Lare, ${ }^{1}$ J. Van de Groep, ${ }^{1}$ \\ J. Garcia de Abajo, ${ }^{3}$ A. Polman, ${ }^{1}$ and H. A. Atwater ${ }^{2}$ \\ ${ }^{1}$ Center for Nanophotonics, FOM-Institute AMOLF, Science Park 104, Amsterdam 1098XG, The Netherlands \\ ${ }^{2}$ Thomas J. Watson Laboratories of Applied Physics, California Institute of Technology, Pasadena, \\ California 91125, USA \\ ${ }^{3}$ IO-CSIC, Serrano 121, Madrid 28006, Spain
}

(Received 8 August 2011; accepted 20 September 2011; published online 13 October 2011)

\begin{abstract}
We have fabricated microphotonic parabolic light directors using two-photon lithography, thin-film processing, and aperture formation by focused ion beam lithography. Optical transmission measurements through upright parabolic directors $22 \mu \mathrm{m}$ high and $10 \mu \mathrm{m}$ in diameter exhibit strong beam directivity with a beam divergence of $5.6^{\circ}$, in reasonable agreement with ray-tracing and full-field electromagnetic simulations. The results indicate the suitability of microphotonic parabolic light directors for producing collimated beams for applications in advanced solar cell and light-emitting diode designs. (C) 2011 American Institute of Physics. [doi:10.1063/1.3648115]
\end{abstract}

Planar micro- and nano-fabrication has enabled the fabrication of a wide array of planar integrated microphotonic components, including mirrors, ${ }^{1-3}$ lenses, ${ }^{4}$ splitters, ${ }^{5}$ array waveguide grating filters, ${ }^{6}$ and other integrated components. ${ }^{7}$ Microphotonic components which control of out-of-plane beam propagation have been designed using planar fabrication methods, such as Bragg mirrors for use in conjunction with vertical cavity surface emitting lasers. ${ }^{8}$ Recently, plasmonic out-of-plane beam steering and focusing structures ${ }^{9-11}$ have also been designed using planar fabrication methods. In many cases, however, the direct fabrication of threedimensional microphotonic components offers greater design flexibility for out-of-plane beam control.

Direct laser write lithography is a promising method to fabricate three dimensional micro- and nano- photonic structures. ${ }^{12}$ It has previously been used for fabrication of three dimensional nanophotonic components such as infrared and optical frequency metamaterials, ${ }^{13}$ but less attention has been devoted to design of three dimension microphotonic components for beam optics. Three dimensional structure fabrication by direct laser writing takes advantage of the ability to expose a well-defined nanoscale voxel using twophoton processes that occur at the waist of a tightly focused infrared beam in a photosensitive resist medium.

In this paper, we report the fabrication of three dimensional microphotonic parabolic beam directors in the form of compound parabolic concentrators via direct laser write lithography. Microphotonic parabolic light directors employ the well-known focusing properties of compound parabolic concentrators, which have previously only been fabricated at the macroscopic scale. ${ }^{14-16}$ Arrays of microphotonic compound parabolic concentrator structures could be used in low-profile format on top of solid-state photonic devices for a wide variety of optoelectronic applications such as collimation of light emitted from light-emitting diodes or improving the efficiency of solar cells by collimating the collected light in order to reduce the entropy changes associated with light emission. ${ }^{17}$

\footnotetext{
${ }^{\text {a) }}$ Author to whom correspondence should be addressed. Electronic mail: jhatwater@gmail.com.
}

In order to create three-dimensional compound parabolic concentrator structures which redirect transmitted light, a Nanoscribe photonic professional two-photon lithography system was used. ${ }^{18}$ The lithography system focuses an expanded $785 \mathrm{~nm}$ laser beam to a diffraction-limited spot at a point where it catalyzes a chemical change in a photoresist (IP-L). This allows the user to write a single voxel instead of a column, allowing three-dimensional structures to be written. During fabrication, a glass substrate coated with optical coupling fluid on one side to facilitate index matching and IP-L two photon negative photoresist on the other is placed at the beam focus and the structure is written in the exposed IP-L by moving the glass substrate. The exposed resist is then developed in 2-propanol, a process during which all unwritten IP-L and all optical coupling fluid is dissolved. After development, only the parabolic structures bonded to the glass substrate remained. The structures are then coated with
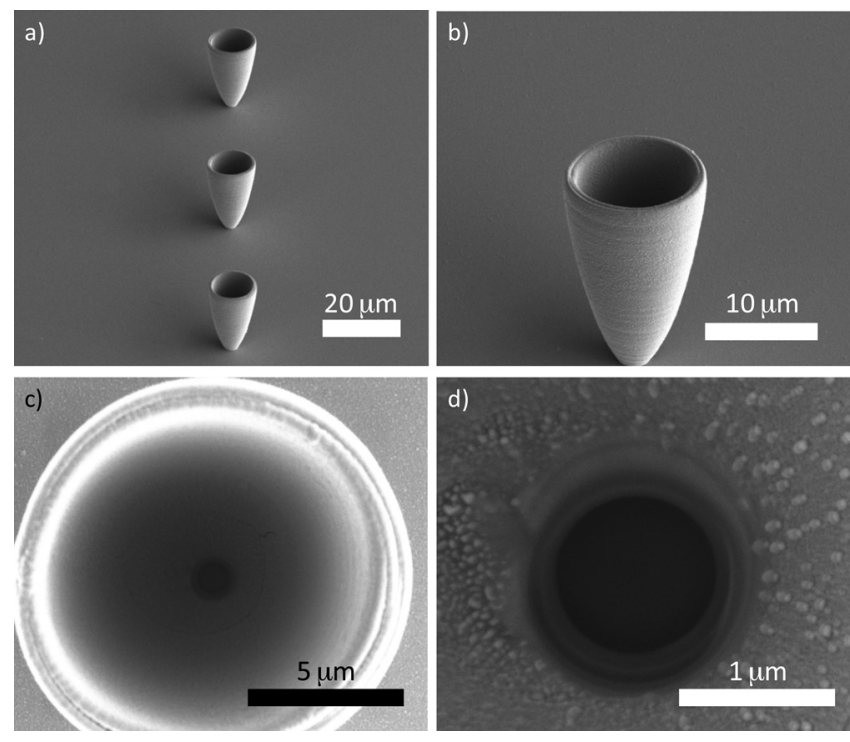

FIG. 1. (a) SEM image of array of parabolic reflectors coated with silver; (b) SEM image of single paraboloid; (c) plan view of single paraboloid with light transmission aperture visible at bottom center; and (d) close up of light aperture etched with a focused ion beam. 

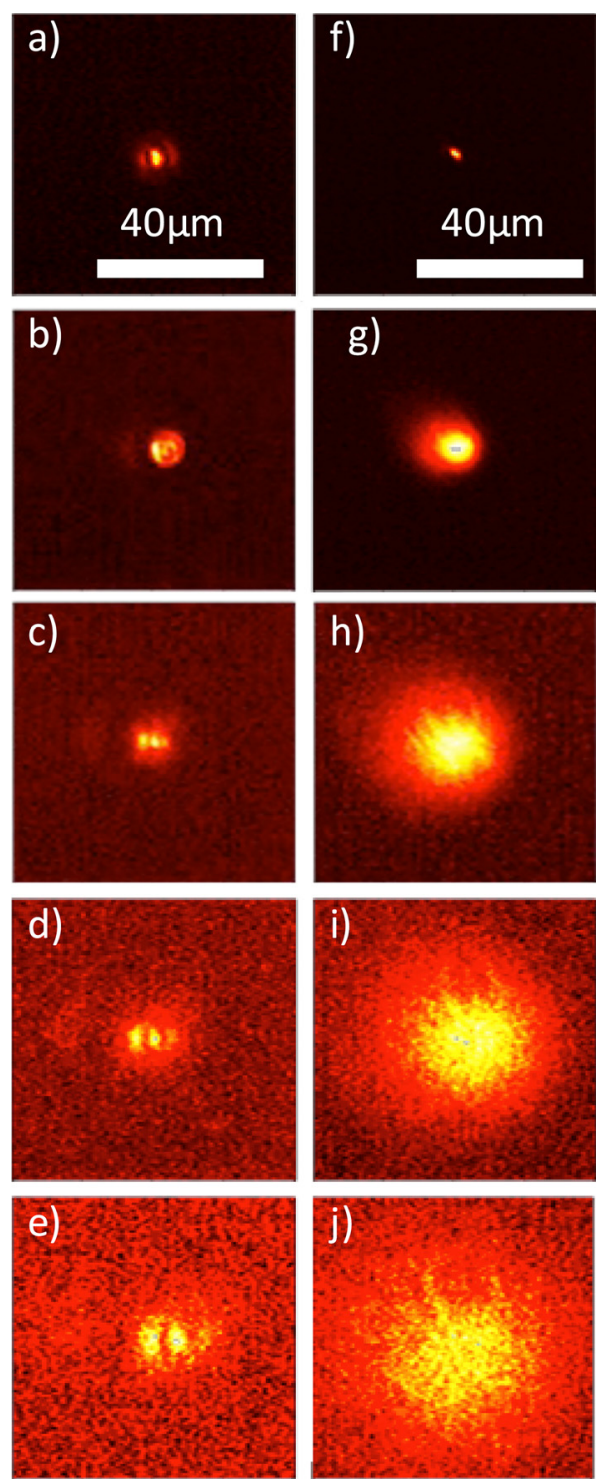

FIG. 2. (Color online) Optical transmission images taken at different heights for parabolic reflector and $1.3 \mu \mathrm{m}$ reference aperture etched into substrate $100 \mu \mathrm{m}$ away with $20 \mathrm{x}$ objective $\mathrm{NA}=0.75$. Images (a)-(e): Paraboloid images with objective at heights $\mathrm{z}=0,25,50,75$, and $100 \mu \mathrm{m}$ above the substrate. Images (f)-(j): Reference aperture images with objective at heights $\mathrm{z}=0,25,50,75$, and $100 \mu \mathrm{m}$ above the substrate.

$20 \mathrm{~nm}$ of chromium and $380 \mathrm{~nm}$ of silver using a plasma sputter coater. Following metal coating, optical apertures with diameters $0.7,1.1$, and $1.3 \mu \mathrm{m}$ were etched into the bottoms of the paraboloids using focused ion beam lithography. The end result is an array of micron-scale, silver-surfaced three-dimensional parabolic structures anchored to a silvercoated substrate at the base of the paraboloids.

During the process of creating microphotonic parabolic structures, several discoveries regarding optimum write techniques were made. Paraboloids written with a wall thickness of 1 voxel (approximately $80-100 \mathrm{~nm}$ ) were found to be unstable during post-exposure processing. To overcome this problem, paraboloids with thicker walls were fabricated by writing five overlapping paraboloids consisting of a center paraboloid and four others offset in the $\mathrm{x}$ and $\mathrm{y}$ directions by $+/-250 \mathrm{~nm}$. The thickness of the metal films used to coat the paraboloids was also found to be an important parameter. In fact, thin Ag films yielded a non-uniform coating of the

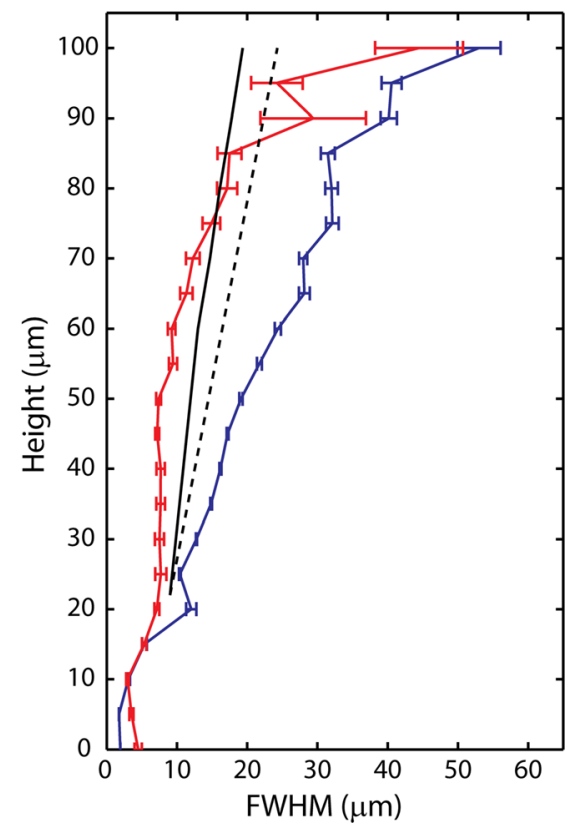

FIG. 3. (Color online) Directivity of transmission for parabolic light directors and reference apertures. FWHM of optical intensity profiles versus objective lens height from transmission images taken at different heights for parabolic reflector (red curve, at left) and reference aperture (blue curve, at right). Dashed line indicates beam divergence half-angle from predictions of ray tracing simulations. Solid line is the divergence half-angle obtained from boundary element method full wave simulations.

parabolas from top to bottom, resulting in significant losses of light due to partial transparency of the metal film at the bottom. Thick Ag films, on the other hand, resulted in increased roughness on the inside and outside walls. The optimal Ag thickness was found to be between 150 and $380 \mathrm{~nm}$.

Figure 1(a) is an electron micrograph of silver-coated $22 \mu \mathrm{m}$ high parabolic light directors spaced by $50 \mu \mathrm{m}$. Figure 1 (b) is an enlarged view of a single paraboloid which illustrates the paraboloid wall thickness resulting from the overlap of exposed voxels in the writing process, visible as concentric rings on the paraboloid, as well as the additional surface roughness associated with the silver coating. Figure 1 (c) is a top view of a single paraboloid that illustrates the $1.3 \mu \mathrm{m}$ wide light aperture visible at the bottom center of the structure. At the rim of the paraboloid, the wall thickness is evident, as are the ripples created by the overlap of exposed voxels during the multiple write process used to thicken the paraboloid walls. Figure 1(d) is an enlarged view of the light aperture which was etched in the bottom of the paraboloid with focused ion beam nanolithography. It indicates that the depth of the light aperture hole is approximately $1 \mu \mathrm{m}$ and extends into the glass layer.

Optical transmission measurements were performed on parabolic light directors and reference apertures in the metalcoated planar substrate with the same size as the ones in the paraboloids. The light source was a broadband supercontinuum laser with approximately uniform intensity in the spectral range between $450 \mathrm{~nm}$ and $1500 \mathrm{~nm}$. A singlewavelength source from an $\mathrm{Ar}$ ion laser operated at $\lambda=514 \mathrm{~nm}$ was also used to study the emission of monochromatic light. Paraboloids and reference apertures were illuminated from below through the glass substrate using a $25 \times$ microscope objective with $\mathrm{NA}=0.5$. The excitation spot was 


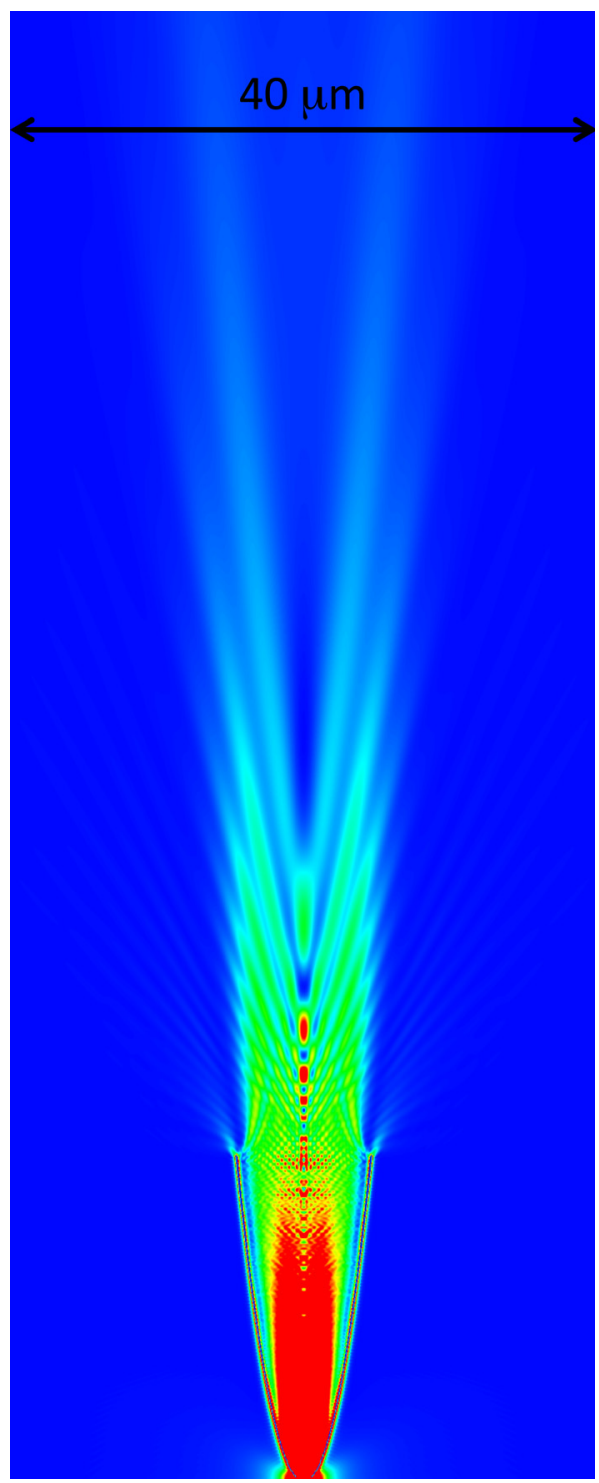

FIG. 4. (Color online) Electric field intensity $|\mathrm{E}(\mathrm{x}, \mathrm{z})|^{2}$ profile at $\lambda=514 \mathrm{~nm}$ for a radial cross section emerging from a $\mathrm{Ag}$ parabola illuminated by a plane wave from bottom. Inset indicates field intensity in vicinity of the parabola with $1.3 \mu \mathrm{m}$ diameter aperture.

defocused to uniformly illuminate both the paraboloids and the reference apertures. Images were taken from the top with a $20 \times$ microscope objective with numerical aperture $\mathrm{NA}=0.75$ and a working distance of $1 \mathrm{~mm}$. Figure 2 shows $514 \mathrm{~nm}$ intensity images taken at heights above the substrate surface ranging from $\mathrm{z}=0-100 \mu \mathrm{m}$, for light transmitted through a paraboloid with a $1.3 \mu \mathrm{m}$ aperture (panels (a)-(e)) and through a reference $1.3 \mu \mathrm{m}$ aperture in the substrate (panels (f)-(j)). Care was taken to record an unsaturated image at the $\mathrm{z}=0$ position in Figs. 2(a) and 2(f), and identical illumination conditions were maintained for Figs. 2(b)-2(e) and $2(\mathrm{~g})-2(\mathrm{j})$ as the distance between the substrate and objective was varied. These images illustrate the ability of paraboloids to collimate light transmitted through the $1.3 \mu \mathrm{m}$ aperture in the bottom into a tight beam in the far field. These results clearly indicate that the microphotonic paraboloid light directors can be used to create collimate beams from micron-scale optical apertures.
Figure 3 shows a plot of the full width half maximum (FWHM) profile obtained from line cuts at the center of the spots shown in Fig. 2 as a function of height above the substrate. The red curve corresponds to the FWHM of the spot imaged above the paraboloids while the blue curve corresponds to the FWHM of the spot imaged above reference aperture. The black dashed line in Figure 3 corresponds to the $5.6^{\circ}$ angular spread of light emitted from the paraboloids predicted by ray tracing calculations. The black solid line represents the emission angle obtained from boundary element method (BEM) calculations. Single-wavelength transmission measurements were also taken at wavelength $\lambda=514 \mathrm{~nm}$ and showed similar results to those seen from the white light source in Figs 2 and 3. The data in Fig. 3 clearly show that the emission of light from the parabola is highly directional and in good qualitative agreement with raytracing and BEM calculations.

Simulations of the electric field for a dipole emission from the parabola were made using the boundary element method. Figure 4 is an image of the calculated electric field intensity $|\mathrm{E}|^{2}$ for emission from a circularly polarized dipole situated at the inner bottom of a parabolic silver mirror. The calculations suggest that the field intensity at the top of the parabola is quite spatial uniform inside the parabola and remains confined in a spatially uniform beam up to a height of approximately $50 \mu \mathrm{m}$, in agreement with the results of Figs. 2(b) and 2(c).

The authors are grateful for technical assistance from $\mathrm{G}$. Vollenbroek and M. Vrucinic. The Caltech researchers are supported by the Light-Matter Interactions Energy Frontier Research Center, an EFRC program of the Office of Science, United States Department of Energy under Grant No. DESC0001293 (EDK and HAA). Researchers of the Center for Nanophotonics at AMOLF are supported by the research program of FOM which is financially supported by NWO.

${ }^{1}$ H. Schenk, P. Dürr, T. Haase, D. Kunze, U. Sobe, H. Lakner, and H. Kück, IEEE J. ST Quantum Electron. 6, 715 (2000).

${ }^{2}$ C.-H. Ji and Y.-K. Kim J. Lightwave Technol. 21, 584 (2003).

${ }^{3}$ W.-H. Juan and S. W. Pang J. Microelectromech. Syst. 7, 213 (1998).

${ }^{4}$ P. Savander, Opt. Lasers Eng. 20, 97 (1994).

${ }^{5}$ J. Leuthold and C. H. Joyner, J. Lightwave Technol. 19, 700 (2001).

${ }^{6}$ S. Suzuki, Y. Inoue, and Y. Ohmori, Electron. Lett. 30, 642 (1994).

${ }^{7}$ T. Tsuchizawa, K. Yamada, H. Fukuda, T. Watanabe, J. Takahashi, M. Takahashi, T. Shoji, E. Tamechika, S. Itabashi, and H. Morita, IEEE J. ST. Quantum Electron. 11, 232 (2005).

${ }^{8}$ G. Hasnain, K. Tai, L. Yang, Y. H. Wang, R. J. Fischer, J. D. Wynn, B. Weir, N. K. Dutta, and A. Y. Cho, IEEE J. Quantum Electron. 27, 1377 (1991).

${ }^{9}$ H. J. Lezec, A. Degiron, E. Devaux, R. A. Linke, L. Martin-Moreno, F. J. Garcia-Vidal, and T. W. Ebbesen, Science 297, 820 (2002).

${ }^{10}$ E. Cubukcu, E. A. Kort, K. B. Crozier, and F. Capasso, Appl. Phys. Lett. 89, 093120 (2006).

${ }^{11}$ Y. C. Jun, K. C. Y. Huang, and M. L. Brongersma, Nat. Commun. 2, 283 (2011).

${ }^{12}$ S. Kawata, H. B. Sun, T. Tanaka, and K. Takada, Nature 412, 697 (2001).

${ }^{13}$ M. S. Rill, C. Plet, M. Thiel, I. Staude, G. von Freymann, S. Linden, and M. Wegener, Nature Mater. 7, 543 (2008).

${ }^{14}$ W. T. Welford and R. Winston. High Collection Nonimaging Optics (Academic, San Diego, 1989).

${ }^{15}$ R. Winston, Sol. Energy 16, 89 (1974).

${ }^{16}$ R. Winston, Appl. Opt. 15, 291 (1976).

${ }^{17}$ E. D. Kosten and H. A. Atwater, Proc. SPIE 8124, 81240F (2011).

${ }^{18}$ N. Anscombe, Nature Photon. 4, 22 (2010). 